\title{
SINGLE POINT BLOW-UP SOLUTIONS TO THE HEAT EQUATION WITH NONLINEAR BOUNDARY CONDITIONS
}

\author{
JUNICHI HARADA
}

Abstract. We study finite blow-up solutions of the heat equation with nonlinear boundary conditions. We provide a sufficient condition for the single point blow-up at the origin and a precise spacial singularity of the blow-up profile.

Mathematics subject classification (2010): 35K20, 35B44.

Keywords and phrases: blow-up, nonlinear boundary condition.

\section{REFERENCES}

[1] M. Abramowitz, I. A. Stegun, Handbook of Mathematical Functions with Formulas, Graphs and Mathematical Tables, Dover Publications, ING., New York, 1965.

[2] D. AMADORI, Unstable blow-up patterns, Differential Integral Equations, 8, 8 (1995), 1977-1996.

[3] J. BRICMONT, A. KupiAinen, Universality in blow-up for nonlinear heat equations, Nonlinearity, 7 , 2 (1994), 539-575.

[4] X. -Y. Chen, H. Matano, Convergence, asymptotic periodicity, and finite-point blow-up in onedimensional semilinear heat equations, J. Differential Equations, 78, 1 (1989), 160-190.

[5] M. CHLEBIK, M. FILA, On the blow-up rate for the heat equation with a nonlinear boundary condition, Math. Methods Appl. Sci., 23, 15 (2000), 1323-2330.

[6] M. ChlebiK, M. Fila, Some recent results on blow-up on the boundary for the heat equation, Evolution equations: existence, regularity and singularities, Banach Center Publ., 52 (2000), 61-71.

[7] S. DejaK, Z. Gang, I. M. Sigal, S. Wang, Blow-up in nonlinear heat equations, Adv. in Appl. Math., 40, 4 (2008), 433-481.

[8] K. Deng, M. Fila, H.A. Levine, On critical exponents for a system of heat equations coupled in the boundary conditions, Acta Math. Univ. Comenian., bf 63 (1994) 169-192.

[9] M. FILA, P. QUITTNER, The blowup rate for the heat equation with a nonlinear boundary condition, Math. Meth. in Appl. Sci., 14 (1991), 197-205.

[10] Y. Giga, R. KoHn, Nondegeneracy of blowup for semilinear heat equations, Comm. Pure Appl. Math., 42 (1989), 845-884.

[11] C. Fermanian Kammerer, F. Merle, H. ZagG, Stability of the blow-up profile of non-linear heat equations from the dynamical system point of view, Math. Ann., 317, 2 (2000), 347-387.

[12] A. Friedman, B. MCLEOD, Blow-up of positive solutions of semilinear heat equations, Indiana Univ. Math. J., 34 (1985), 425-447.

[13] J. HARADA, Blow-up behavior of solutions to the heat equation with nonlinear boundary conditions, preprint.

[14] M. A. Herrero, J. J. L. Velázquez, Flat blow-up in one-dimensional semilinear heat equations, Differential Integral Equations, 5, 5 (1992), 973-997.

[15] M. A. Herrero, J. J. L. Velázquez, Blow-up profiles in one-dimensional semilinear parabolic problems, Comm. Partial Differential Equations, 17, 1-2 (1992), 205-219.

[16] M. A. Herrero, J. J. L. VelázqueZ, Blow-up behavior of one-dimensional semilinear parabolic equations, Ann. Inst. Henri Poincare, 10, 2 (1993), 131-189.

[17] S. Hitomatsu, S. Moriguchi, K. Udagawa, Mathematical Formula III, Iwanamishoten, 1987 (in Japanese). 
[18] B. HU, Nondegeneracy and single-point-blowup for solution of the heat equation with a nonlinear boundary condition, J. Math. Sci. Univ. Tokyo, 1, 2 (1994), 251-276.

[19] B. HU, H. -M. YIN, The profile near blowup time for solution of the heat equation with a nonlinear boundary condition, Trans. Am. Math. Soc., 346 (1994), 117-135.

[20] F. MerLe, Solution of a nonlinear heat equation with arbitrarily given blow-up points, Comm. Pure Appl. Math., 45, 3 (1992), 263-300.

[21] F. Merle, H. ZAGG, Stability of the blow-up profile for equations of the type $u_{t}=\Delta u+|u|^{p-1} u$, Duke Math. J., 86, 1 (1997) 143-195.

[22] F. MERLE, H. ZAGG, Refined uniform estimates at blow-up and application for nonlinear heat equations, Geom. Funct. Anal., 8, 6 (1998), 1043-1085.

[23] J. J. L. VeLÁzQUEZ, Higher dimensional blow up for semilinear parabolic equations, Commun. in Partial Differential Equations, 17, 9-10 (1992), 1567-1596. 